\title{
Feedback in Separated Flows Over Symmetric Airfoils
}

H. M. Atassi

Lewis Research Center

Cleveland, Ohio

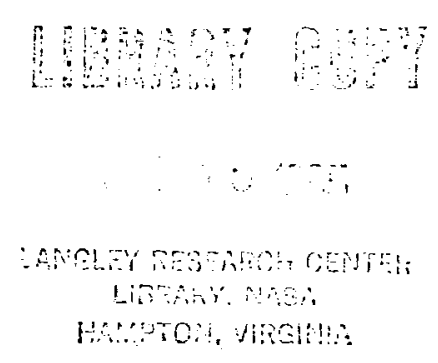

Prepared for the

Ninth Aeroacoustics Conference

sponsored by the American Institute of Aeronautics and Astronautics Williamsburg, Virginia, October 15-17, 1984 


\author{
H. M. Atass $j *$ \\ National Aeronautics and Space Administration \\ Lewis Research Center \\ Cleveland, Ohio 44135
}

\section{Abstract}

It is shown that for a flow over an airfoil with laminar separation, a feedback cycle may exist whereby a Kelvin-Helmholtz instability wave emanating from the separation point on the airfoil surface grows along the shear layer and is diffracted as it interacts with the sharp trailing edge of the airfoil causing acoustic radiation which in turn propagates upstream and regenerates the initial instability wave. The analysis is restricted to the high frequency limit. Solutions to the boundaryvalue problem are obtained using the slowly varying approximation and the method of matched asymptotic expansions. It is shown that resonant solutions exist for certain discrete values of the Reynolds and Strouhal numbers. The results are discussed and compared with available data.

\section{Introduction}

Recent experiments by Paterson et ar. 1 in which NACA 0012 and 0018 airfoils were placed in flows at Reynolds numbers of $4 \times 10^{5}$ to $2.8 \times 10^{6}$ revealed the existence of well defined discrete tones in the noise spectra. The measured variation of the tone frequency versus the flow velocity indicated a frequency dependence on the velocity to the 0.8 power with an occasional jump in frequency leading to a ladder structure behavior. At Reynolds numbers and angles of attack for which the viscous boundary-layer was turbulent on both surfaces of the airfoil, the discrete tones were undetectable above turbulent boundary-layer noise. Paterson et al. attributed the generation of the tones to vortex shedding. Tam ${ }^{2}$ arguing that these tones are unrelated to vortex shedding, proposed a qualitative acoustic feedback mechanism between the near wake and the trailing edge. Fink ${ }^{3}$ argued that the noise radiated was a direct result of hydrodynamic instabilities in the laminar boundary-layer. He recovered the boardband frequency dependence on velocity to the 1.5 power. However, his analysis cannot explain the existence of the discrete tones, their ladder structure and their 0.8 power dependence on velocity. Finally, more recent measyrements were carried out by Arbey and Bataille on NACA 0012 airfoils at zero angle of attack. Their results are in accord with those of Paterson, et al. Arbey and Bataille used a combination of Tam's and Fink's suggestions to correlate their data. They essentially assumed that TollmienSchlichting instability waves propagating downstream in the laminar boundary-layer are diffracted by the trailing edge and thus produce acoustic waves which are "received" at the minimum pressure point of the airfoil and regenerate the initial instability waves.

\#Addison P. Rothrock Distinguished Scientist. Permanent address: University of Notre Dame, Notre Dame, Indiana 46556.
The mechanism proposed by Arbey and Bataille is, however, incompatible with the following two observations. First, the generation of TollmienSchlichting waves in flows over not too thin bodies by external acoustic forcing is either strongly related to the magnitude of the local mean pressure gradient of the airfoil as shown by Goldstein, 5 or to the gradient of the unsteady forcing in the case of a localized source as shown by Nishioka and Morkovin. 6 Second, although the condition of the boundary-layer is not discussed in Refs. 1 and 4, the flow characteristics of a NACA 0012 airfoit have been extensively investigated. Mccroskey carried out experimental and analytical investigations of such airfoils for a Reynolds number range of $10^{5}$ to $10^{6}$ and gave the position of the laminar separation point for angles of attack a ranging from $0^{\circ}$ to $8^{\circ}$. At $a=0^{\circ}$, the separation point was located at 0.70 chord iength from the leading edge. Thus the boundary-layer is separated in both Paterson's, et al. and Arbey and Bataille's experiments.

Separated flows over airfoils at low and moderate Reynolds numbers are known to exhibit large scale coherent motions which are associated with fluctuating lift and broad band acoustic radiation. The experiments in Refs. 1 and 4 then show that under certain conditions the sound spectrum of such flows also exhibits well-defined discrete tones. These tones are almost certainly the result of a self-sustainable oscillatory flow phenomenon. We therefore conjecture that a feedback cycle may exist in such separated flows whereby a spatially growing Kelvin-Helmholtz instability wave emanating from the separation point on the airfoil surface propagates downstream and is diffracted as it interacts with the sharp trailing edge of the airfoil causing acoustic radiation which in turn propagates upstream and regenerates the initial instability wave.

The present conjecture is essentially based on the following two observations. First, it is now well known 8 that when a shear layer interacts with a sharp edge, it produces a pressure wave that propagates upstream. Second, as recently shown by Goldstein, 9 an external forcing will generate a spatially growing Kelvin-Helmholtz instability wave at the separation point of a flow that separates from a smooth surface.

To simplify the analysis we only consider the case of a symmetric airfoil at zero angle of attack (Fig. 1). The general case is treated in Ref. 10 . We assume small-amplitude time-dependent harmonic disturbances about the steady separated flow. At high Reynolds numbers, the steady flow is given to leading order in Reynolds, number by the Kirchhoff free streamline solution. 11,12 Moreover, because of the inhibiting effect of the nearby airfoil surface, the most rapidly growing instability waves have wave iengths that are small compared to the airfoil chord. The analysis is therefore restricted to the high frequency limit. 
In Section II we develop the general scaing of the problem and derive the governing equations and boundary conditions for the boundary value problem. To construct the general solution we use the siowly varying approximation (i.e., the method of multiple scales) and the method of matched asymptotic expansions. The flow domain is divided in two regions. First, a region surrounding the separation point where we use Goldstein's eigensolution representing a spatially growing KelvinHelmholtz instability wave emanating from the separation point. The second region surrounds the trailing edge. In Section III, we construct the solution in the trailing edge region by the wienerHopf method. In Section IV, the two solutions are matched in an overlap region and furthermore the trailing edge solution provides the hydrodynamic forcing for the eigensolution emanating from the separation point.

Finally, in Section $V$ the results are discussed and compared with those obtained from experiments.

\section{Formulation}

We consider an airfoil of infinite span and sharp trailing edge 0 placed in a uniform incompressible flow with upstream velocity $U_{\infty}$. The airfoil chord length is $c$. The flow Reynolds number $\operatorname{Re}=\mathrm{CU}_{\infty} / v$, where $v$ is the kinematic viscosity, is assumed to be large. We further suppose the flow to be separated on the airfoil surface. The flow is then given to leading order in Reynolds number by the Kirchhoff free streamline solution. Figure 2 shows the configuration of the separated flow around the airfoil. For convenience the flow is divided in four regions. The airfoil thickness $\delta$ is assumed to be small. Then, according to airfoil theory, the equations for the airfoif surface and the free streamline are respectively

$$
y^{\star}= \pm \delta H_{b}\left(x^{\star}\right) \text {, }
$$

and

$$
y^{*}= \pm \delta H_{S}\left(x^{*}\right)
$$

where $\left(x^{\star}, y^{\star}\right)$ represent the coordinates nondimensionalized with respect to $c$. $H_{b}$ and $H_{s}$ are then $0(1)$.

We now assume the flow to have a harmonic motion whose amplitude is characterized by a small parameter $a_{0}$ and whose frequency is $\omega$. The velocity of the harmonic motion is assumed to be small compared to $U_{\infty}$. Futhermore we assume the reciprocal of the Strouhal number $S$

$$
\varepsilon=\frac{1}{S} \times \frac{U_{\infty}}{C_{\omega}}<<1 \text {. }
$$

In what follows we assume $\delta=O(\varepsilon)$ and we nondimensionalize the time $t$ by $\omega^{-1}$, the velocities by $U$, the pressure by $\rho U^{2}$ and the lengths by $U_{\infty} / \omega$. "Thus the coordinates of a point $\vec{x}=(x, y)$ will be related to $\left(x^{\star}, y^{\star}\right)$ by $x^{\star}=$ ex and $y^{*}=\varepsilon y$.

Since the vorticity is confined only to layers along the separation lines, the fluid motion is irrotational. The velocity potential can then be expanded as

$$
\begin{aligned}
\theta_{j}(\vec{x}, t)=\phi_{j}^{(0)}(\vec{x})+a_{0}{ }_{j}^{(1)}(\vec{x}) e^{-i t} & \\
j & =1,2,3,4
\end{aligned}
$$

where ${ }_{j}^{(0)}(\vec{x})$ is the velocity potential in region $j$ (Fig. 2) corresponding to the Kirchhoff solution, and $\phi_{j}^{(1)}(\vec{x}) e^{-i t}$ is the velocity potential of the harmonic motion in region $j . i=\sqrt{-1}$. The velocity $\vec{u}_{j}(\vec{x})+a_{0} \vec{u}_{j}(\vec{x}) e^{-i t}$ can then be written as

$$
\nabla_{j}=\nabla \phi_{j}^{(0)}
$$

and

$$
\vec{u}_{j}=\nabla \phi_{j}^{(1)} \text {. }
$$
In the separated region $(j=2,3) 0_{j}=0$, and along
the free streamline $\left|0_{j}\right|=1$.

The unsteady flow is solenoidal and irrotational and therefore the governing equations are

$$
\nabla^{2} \Phi_{j}^{(1)}=0 \text {. }
$$

The boundary conditions along the free streamlines are identical to those derived by Goldstein 9 (Eqs. 2.16 to 2.18). We further note that EqS. (1) and (2) depend solely on $x^{*}$. Therefore, in order to account for the effects of a diverging mean flow we use the method of multiple scales. We now introduce

$$
\Delta=\frac{\delta}{\varepsilon}\left[H_{s}-H_{b}\right]
$$

Then $d \Delta / d x=0(\delta)$, and $d\left(0_{j} \cdot 0_{j}\right) / d x=0(\delta c)$ along a free streamline. This suggests that, except in the neighborhood of the separation point, ${ }_{j}(1)$
has an expansion of the form

$$
\phi_{j}^{(1)}=\varphi_{j}^{(0)}\left(x, y ; x^{\star}\right)+\delta \varphi_{j}^{(1)}\left(x, y ; x^{\star}\right)+\ldots
$$
The equations and boundary conditions for $\varphi_{j}^{(0)}$
are then readily obtained

$$
\nabla^{2} \varphi_{j}^{(0)}=0 \quad j=1,2,3,4
$$

$$
\begin{aligned}
& \left(1+i \frac{\partial}{\partial x}\right) \varphi_{k}^{(0)}=\varphi_{k \pm 1}^{(0)} \\
& \left.\left(1+i \frac{\partial}{\partial x}\right) \frac{\partial}{\partial y} \varphi_{k \pm 1}^{(0)}=\frac{\partial}{\partial y} \varphi_{k}^{(0)}\right) \\
& \text { at } y= \pm \frac{\delta}{\varepsilon} H_{S}\left(x^{*}\right)
\end{aligned}
$$




$$
\begin{aligned}
& \frac{\partial}{\partial y} \varphi_{k}^{(0)}=0 \\
& \text { at } y= \pm \frac{\delta}{\varepsilon} H_{b}\left(x^{\star}\right) \text {. } \\
& \frac{\partial}{\partial y} \varphi_{k \pm 1}^{(0)}=0 \\
& \text { In Eqs. (11) and (12) }+ \text { must be taken for } k=1 \\
& \text { and - for } k=4 \text {. }
\end{aligned}
$$

In order to construct the general solution to this boundary-value problem, we use the method of matched asymptotic expansions. First we consider the regions surrounding the separation points $S$ and $S^{\prime}$. Here we use Goldstein's eigensolutions which represent spatially growing Kelvin-Helmholtz instability waves emanating from $S$ and $S^{\prime}$. Second, we consider a trailing edge region whose length is such that $|x| \gg 1$ but $\left|x{ }^{*}\right| \ll 1$. The trailing edge solution must match onto the KelvinHelmholtz eigensolutions in some overlap domain and moreover it must provide the hydrodynamic forcing for the eigensolutions.

\section{Solution in the Trailing Edge Region}

We consider a region surrounding the trailing edge 0 whose length is such that $|x| \gg 1$ but $\left|x{ }^{*}\right|<1$. To leading order in the $(x, y)$ coordinates this region is reduced to a semi-infinite plane extending to the left and two vortex sheets located at a distance $\Delta_{0}$ from the semi-infinite plane (Fig. 3). The solution to the boundary-value problem (Eqs. (10) to (12)) will be obtained by the Wiener-Hopf technique. However, we first give the downstream asymptotic form of the solution.

Far downstream we have two vortex sheets at a distance $2 \Delta_{0}$ from each other. Under these conditions we have

$$
\varphi_{1, \infty}^{(0)}=A^{+} e^{-\alpha^{+}\left(y-\Delta_{0}\right)} e^{i \alpha^{+} x}
$$

$$
\begin{aligned}
\varphi_{2, \infty}^{(0)}=\varphi_{3, \infty}^{(0)} & =A^{+}\left\{\left(1-\alpha^{+}\right) \cosh \alpha^{+}\left(y-\Delta_{0}\right)\right. \\
& \left.-\frac{1}{1-\alpha^{+}} \sinh \alpha^{+}\left(y-\Delta_{0}\right)\right\} e^{i \alpha{ }^{+} x}
\end{aligned}
$$

$=A^{+}\left\{\left[\cosh 2 a^{+} \Delta_{0}+\frac{\sinh 2 \alpha^{+} \Delta_{0}}{\left(1-a^{+}\right)^{2}}\right] e^{\alpha^{+}\left(y+\Delta_{0}\right)}\right\} e^{i \alpha^{+} x}$

where

$$
\varphi_{j, \infty}^{(0)}=\varphi_{j}^{(0)} \text { as } x+\infty, j=1,2,3,4
$$

$\mathrm{A}^{+}$is a constant and $\alpha^{+}$is solution to the characteristic equation

$$
(\alpha-1)^{2} \sinh \alpha \Delta_{0}+\cosh \alpha \Delta_{0}=0,
$$

and then is denoted $a_{s}^{+}$since it represents the wave number of a symetric or varicose mode, or it is solution to

$$
(\alpha-1)^{2} \cosh \alpha \Delta_{0}+\sinh \alpha \Delta_{0}=0,
$$

and then is denoted $a_{a}^{+}$since it corresponds to an antisymetric or sinuous mode. In both cases $a^{+}$should correspond to a spatially growing wave, i.e.,

$$
I_{m}\left\{\alpha^{+}\right\}<0
$$

Equations (13) to (15) show that the solution to Eqs. (10) to (12) will be unbounded for growing instability waves. We therefore introduce

$$
\dot{\varphi}_{j}=\varphi_{j}^{(0)}-\varphi_{j, \infty}^{(0)} .
$$

${ }_{j}$ will be bounded everywhere except maybe at the trajling edge.

We now introduce the Fourier transforms of $\varphi_{j}$

$$
\begin{aligned}
& \tilde{\varphi}_{j}(\alpha, y)=\frac{1}{\sqrt{2 \pi}} \int_{-\infty}^{+\infty} \varphi_{j}(x, y) e^{i \alpha x} d x \\
& \tilde{\varphi}_{j}^{+}(\alpha, y)=\frac{1}{\sqrt{2 \pi}} \int_{0}^{\infty} \varphi_{j}(x, y) e^{i \alpha x} d x \\
& \tilde{\varphi}_{j}(\alpha, y)=\frac{1}{\sqrt{2 \pi}} \int_{-\infty}^{0} \varphi_{j}(x, y) e^{i \alpha x} d x
\end{aligned}
$$
into

The governing equation is then transformed

$$
\frac{d^{2} \tilde{\varphi}_{j}}{d y^{2}}-a^{2} \tilde{\varphi}_{j}=0 .
$$

Noting that regions 1 and 4 extend to infinity, the solutions to Eq. (24) must then be of the form $\exp \{\bar{F}|a| y\}$. However, since we will construct a solution by the Wiener-Hopf technique, we need to extend the definition of these solutions to a common strip in the complex $\alpha-p$ lane where they are both analytic. This is done by taking solutions of the form

$$
\tilde{\varphi}_{j}=A_{j} e^{\gamma y}+B_{j} e^{-\gamma y}
$$

where

$$
r=\left(\alpha^{2}+\varepsilon_{1}^{2}\right)^{1 / 2}
$$

and $E_{1}$ is a small positive quantity. The branch cuts for $\gamma$ are shown in Fig. 4. Substituting Eq. (25) into the boundary conditions derived from EqS. (11) and (12), we obtain after considerable reduction 


$$
\tilde{\varphi}_{2}^{+1}=\gamma K(\alpha)\left(\tilde{\varphi_{3}}-\tilde{\varphi_{2}}\right)+\tilde{\varphi}_{2, \infty}^{+1}
$$

where

$$
\begin{gathered}
K(\alpha)=\frac{f\left(\alpha, \Delta_{0}\right)}{g\left(\alpha, \Delta_{0}\right)}, \\
f(\alpha, \Delta) \equiv(1+\alpha)^{2} \sinh \gamma \Delta+\cosh \gamma \Delta, \\
g(\alpha, \Delta) \equiv(1+\alpha)^{2} \cosh \gamma \Delta+\sinh \gamma \Delta,
\end{gathered}
$$

and

$$
\stackrel{p+1}{\varphi, \infty}_{2,}=\frac{D}{a+a^{+}}
$$

is the Fourier transform of $\varphi_{2, \infty}(0)^{\prime}$ with

$$
D=\frac{i A^{+} \alpha^{+}}{\sqrt{2 \pi}\left(1-\alpha^{+}\right)} f\left(-\alpha^{+}, \Delta_{0}\right) \text {. }
$$

$K(a)$ is regular and nonzero in the strip $-\varepsilon 1<\tau<\varepsilon 1,-\infty<\sigma<\infty$, and $K(\alpha) \rightarrow 1$ as $\sigma \rightarrow \pm \infty$ in the strip, then we can write

$$
K(\alpha)=\frac{K_{-}(\alpha)}{K_{+}(\alpha)}
$$

where $K_{+}(\alpha)$ and $K_{-}(\alpha)$ are regular, bounded and nonzero in $\tau>-\varepsilon \varepsilon_{1}$ and $\tau<\varepsilon$, respectively. Substituting Eq. (33) into Eq. (27), rearranging the terms and introducing the functions $G_{+}(\alpha)$ analytic for $\tau>-\varepsilon_{1}$ and $G_{-}(a)$ analytic for $\tau<\varepsilon_{1}$ and whose jump along the $\sigma$-axis is

$$
\frac{D K_{+}(\alpha)}{(\alpha+\alpha)\left(\alpha+\varepsilon_{1} i\right)^{1 / 2}} \text {. }
$$

we finally obtain

$$
\begin{aligned}
J(\alpha) & =\frac{\tilde{\varphi}_{2}^{+} K_{+}(\alpha)}{\left(\alpha+\varepsilon_{1} i\right)^{1 / 2}}-G_{+}(\alpha) \\
& =K_{-}(\alpha)\left(\alpha-\varepsilon_{1} i\right)^{1 / 2}\left(\tilde{\varphi_{3}}-\tilde{\varphi_{2}}\right)-G_{-}(\alpha) .
\end{aligned}
$$

If we further impose the condition $\varphi^{\prime}(0) \sim x^{-1 / 2}$ at the edge $x=0$, we can show using Liouvilie's theorem that $J(a)$ must identically be zero. We finally arrive at the following expression

$$
\tilde{\varphi}_{2}^{\prime}(\alpha, y)=-\frac{D}{\alpha+\alpha^{+}}\left(\frac{a}{-\alpha^{+}}\right)^{1 / 2} \frac{K_{+}\left(-\alpha^{+}\right)}{K_{+}(\alpha)} \frac{f\left(\alpha, \Delta_{0}-y\right)}{f\left(\alpha, \Delta_{0}\right)}
$$

and a similar expression for $\tilde{\varphi}_{3}^{\prime}(\alpha, y)$.

Taking the inverse Fourier transform of Eq. (35), we get

$$
\varphi_{j}^{\prime}=\frac{-D K_{+}\left(-\alpha^{+}\right)}{\sqrt{2 \pi}\left(-\alpha^{+}\right)^{1 / 2}} \int_{-\infty}^{+\infty} \frac{\sqrt{\alpha} f\left(\alpha, \Delta_{0} \mp y\right) e^{-i \alpha x}}{K_{+}(\alpha) f\left(\alpha, \Delta_{0}\right)\left(\alpha+a^{+}\right)} d x
$$

where $F$ corresponds to $j=2$ and $j=3$, respectively.

- We now examine the expression for $D$ (Eq. (32)). If $a_{+}^{+}$corresponds to a symmetric mode, i.e., $\alpha^{+}=\alpha_{s}^{+}$, then, $D\left(\alpha_{s}^{+}\right)=0$. Thus as expected instability waves with downstream symmetric modes propagate through the trailing edge region without any modification and consequently they do not produce any scattered hydrodynamic field. Hence,

$$
\varphi_{j, s}^{(0)^{\prime}}=\frac{A_{s^{+} a_{s}^{+}}^{+}}{a_{s}^{+}-1} \frac{\sinh a_{s}^{+} y}{\sinh a_{s}^{+} \Delta_{0}} e^{i a_{s}^{+} x} j=2,3 .
$$

We now examine Eq. (36) for $a^{+}$corresponding to an ant isymmetric mode, i.e., $\alpha^{+}=\alpha_{\mathrm{a}}^{+}$. It can be shown that $f\left(a, \Delta_{0}\right)$ has an infinite number of zeros and that the zero, say, $-a_{s}$ whose imaginary part has the smallest absolute value is such that $\operatorname{Re}\left(a_{s}\right)>0$ and $\operatorname{Im}\left(a_{s}\right)<0$. Thus as corresponds to a growing instability wave propagating downstream. The other zeros of $f\left(\alpha, \Delta_{0}\right)$ are of the form

$$
a_{n}^{ \pm}= \pm\left(\sigma_{n}+i \tau_{n}\right)
$$

where both $\sigma_{n}$ and $\tau_{n}$ are positive.

In order to evaluate Eq. (36) for $x<0$, we close the contour in the upper half of the complex $a-p l a n e$. Then using Cauchy's formula and theorem to evaluate the contour integral and substituting the result and Eq. (14) into Eq. (20) we obtain

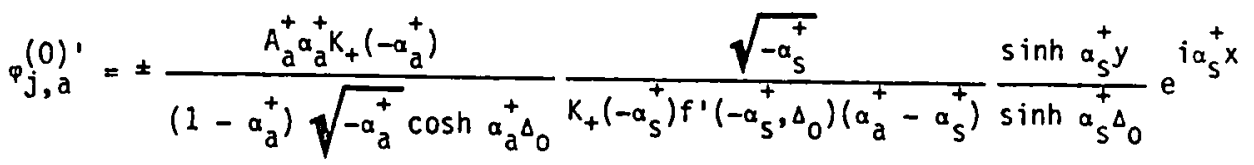

$$
\begin{aligned}
& +\sum_{n=1}^{\infty} \frac{\sqrt{a_{n}^{+}}}{k_{+}\left(a_{n}^{+}\right) f^{+}\left(a_{n}^{+}, \Delta_{0}\right)\left(a_{a}^{+}+a_{n}^{+}\right)} \frac{\sinh a_{n}^{+} y}{\sinh a_{n}^{+} \Delta_{0}} e^{-i a_{n}^{+} x}+\frac{\sqrt{i}}{\pi} \int_{0}^{\infty} \frac{\sqrt{\tau} e^{\tau x}\left(i_{\tau}+1\right)^{2} \sin \tau y}{k^{+}\left(i_{\tau}\right)\left(i_{\tau}+a_{a}^{+}\right)\left[\left(i_{\tau}+1\right)^{2} \sin ^{2} \tau \Delta_{0}+\cos ^{2} \tau \Delta_{0}\right]} d \tau
\end{aligned}
$$


where \pm corresponds to $j=2$ and $j=3$. respectively.

The first term in Eq. (39) represents a growing instability wave propagating downstream. The second term represents an infinite number of trapped acoustic modes propagating upstream and decaying. Finally the third term which results from the branch cut integral represents the scattered hydrodynamic field. It is important to note that $\varphi_{2, a}(0)^{\prime}$ and $\varphi_{3, a}^{(0)^{\prime}}$ are in phase at symmetric points $\pm y$ in regions 3 and 4 , respectively.

IV. Matching with the Instability Waves Emanating From the Separation Points

We now turn out attention to the solution of the boundary-value problem (Eqs. (10) to (12)) in a region $0(1)$ surrounding the separation point. Such a solution was given by Goldstein ${ }^{9}$ (Eq. 45). For $|x| \gg 1$ but $\left|x^{\star}\right| \ll 1$, this solution can be written as

$\varphi_{j}(0)^{\prime}$

$$
\begin{gathered}
=-c_{j} \sqrt{3\left(\frac{d \alpha_{s}}{d \Delta}\right)_{0}} \sinh \alpha_{s_{0}} y e^{i \int_{s}^{0} a_{s}\left(x^{\star}\right) d x} e^{i \alpha_{s 0} x} \\
j=2,3
\end{gathered}
$$

where $C_{j}$ are the coupling coefficients with the external forcing, $a_{s}$ is solution to Eq. (17) with $\Delta_{0}$ replaced by $\Delta, a_{s 0}=\alpha_{s}(0)$ and the subscript - denotes a quantity evaluated at the trailing edge. The expressions for $C_{j}$ which are proportional to some inverse power of the Reynolds number and depend on the airfoil geometry and the Strouhal number, were derived in Refs. 9 and 14.

In the present problem the external forcing is the scattered hydrodynamic field which can be calculated from the branch cut integral in Eq. (39). Thus, for large $|x|$, the pressure gradient at the separation point is

$$
P_{j}^{\prime}=\left(\frac{\partial P_{j, a}^{(0)}}{\partial x}\right)_{s}
$$

$$
\begin{aligned}
& =\mp \frac{3}{\sqrt[4]{\pi i}} \frac{A_{a}^{+} K_{+}\left(-a_{a}^{+}\right)}{\left(1-a_{a}^{+}\right) \sqrt{-a_{a}^{+}} K_{+}(0) \cosh a_{a}^{+} \Delta_{0}} \\
& \times\left\{\frac{1}{|x|^{3 / 2}}+\ldots \mid\right.
\end{aligned}
$$

where $p_{j, a}(0)$ denotes the pressure corresponding to $\varphi_{j, a}^{(0)}$ and the subscript $s$ denotes a quantity evaluated at the separation point. Note that $P_{2}$ and $P_{3}$ have a phase difference equal to $\pi$. Since $C_{j}$ is proportional to $P_{j, \Phi_{2}^{\prime}}^{\prime}(0)$ and $\varphi_{3}^{\prime}(0)$ defined by Eq. (40), have no phase difference at symmetric points into regions 2 and 3 . On the other hand, $\varphi_{j, s}^{(0)}$ ' represents waves with a phase difference equal to $\pi$ at symetric points in regions 2 and 3 . Therefore $\varphi_{\mathrm{j}, \mathrm{s}}^{(0)^{\prime}}$ cannot match with the $\varphi_{j}^{(0)^{\prime}}$ and $A_{S}^{+}$must vanish. Thus

$$
\varphi_{j, S}^{(0)^{\prime}}=0
$$

Matching the upstream expansion of Eq. (39) with Eq. (40) gives

$c \sqrt{3\left(\frac{d a_{s}}{d \Delta}\right)_{0} e^{i} \int_{x_{s}}^{0} a_{s}\left(x^{\star}\right) d x}=\mp A_{a}^{+} \frac{a_{a^{+} k_{+}\left(-a_{a}^{+}\right)}^{\left(1-a_{a}^{+}\right) k_{+}\left(-a_{s}^{+}\right)}}{\left(1-a^{+}\right.}$ $\times\left(\frac{a_{s}^{+}}{a_{a}^{+}}\right)^{1 / 2} \frac{1}{f^{\prime}\left(-a_{s}^{+}, \Delta_{0}\right)\left(a_{a}^{+}-a_{s}^{+}\right) \cosh \alpha_{a}^{+} \Delta_{0} \sinh a_{s}^{+} \Delta_{0}}$.

where $\Delta_{0}$ is the value of $\Delta$ at the origin.

We now examine Eq. (43) and note that for $\Delta=0(1), \alpha_{s_{+}}=0(1)$ and that $C_{j}$ is also proportional to $A_{\mathrm{a}}^{+}$. Moreover, $\delta_{j}$ is proportional to some inverse power of Re. 9,14 By matching the leading order terms in Eq. (43) we find

$$
S=\frac{1}{\varepsilon} \sim \ln \operatorname{Re} \text {. }
$$

Four important results for the remainder of our analysis can be drawn from Eq. (44). First, it justifies our modeling of the free-shear layer by a vortex sheet. Second, it insures the validity of Goldstein's solutions (Eq. 40) for the instability waves emanating from the separation points. Third, it implies that the flow in the "triple deck" regions surcounding the separation points is quasisteady. 15 And fourth, it determines the partiguiar expressions for the coupling coefficients $C_{j} .9$ Therefore, from Ref. 14, Eq. 4.8

$$
c_{j}=\frac{-15 i}{16 \sqrt{6}} \frac{\hat{f}(0)}{\hat{f}^{\prime}(0)} \sigma^{1 / 4} c^{\star^{2}} \varepsilon^{1 / 2} \varepsilon_{0} P_{j}^{\prime}
$$

where

$$
\varepsilon_{0}=(\operatorname{Re})^{-1 / 8}
$$

and $c^{\star}$ is a coefficient in the expansion of the free streamline equation (Eq. (2)) in terms of the $\left(x^{\star}, y^{\star}\right)$ coordinates (Fig. 2) for $x^{\star}+0^{+}$

$$
y^{\star}=a^{\star} x^{\star^{3 / 2}}+b^{\star} x^{\star^{2}}+c^{\star} x^{\star^{5 / 2}}+0\left(x^{\star^{3}}\right)
$$

where

$$
a^{\star}=\hat{f}(0) \varepsilon_{0} \sigma^{9 / 8} .
$$

The function $\hat{f}$ is defined in Ref. 9 (Eq. 8,2 ). $f(0)=2 / 3(0.44)$, was calculated by Smith. 11 $f^{\prime}(0)$ is the derivative of $f$ for a quasi-steady "triple deck" flow with a nonzero wall boundary 
condition. Finally $\sigma$ is the scaled skin friction just ahead of the separation point. For a thin airfoil, $\sigma$ can be approximated by Blasjus expression for the flat plate, $\sigma=0.332 / \sqrt{x_{0}}$ where $x_{0}^{\star}$ is the distance of the separation point from the leading edge.

With the expression of $C_{j}$ given by Eq. (45), it is now possible to examine the order of certain terms in Eq. (43). The location of the separation point can be inferred from Sychev 11

$$
x_{0}^{\star}=\left(x_{0}^{\star}\right)_{B P}+0\left(\varepsilon_{0}^{1 / 2}\right)
$$

where $\left(x_{0}^{\star}\right)$ denotes the position of the Brillouin point. For ${ }^{B P}$ slender profite Cheng and $S$ mith ${ }^{16}$ used Brodetsky's ${ }^{17}$ free streamline solution for approximately elliptic profiles to show that

$$
\left(x_{0}^{*}\right)_{B P}=O(\varepsilon)
$$

We also note that even though EqS. (39) and (40) are the zero-order terms in the expansion (Eq. (9)), they both contain a phase correction to $0(\varepsilon)$. Our analysis will then be consistent if we keep in Eq. (43) all terms up to $O(1)$. Substituting Eq. (41) jnto Eq. $(45)$ and then eliminating $C_{j}$
from Eqs. (43) and (45) we obtain by equating the amplitudes and phases of both sides of the resulting equation

$$
\begin{gathered}
\frac{1}{\varepsilon_{0}}=A \varepsilon^{15 / 8} \exp \left\{-\bar{a}_{i} e^{\star} / \varepsilon\right\} \\
\frac{e^{\star} \bar{a}_{r}}{\varepsilon}=\psi+2 \pi n
\end{gathered}
$$

where $\bar{a}_{r}$ and $\bar{\alpha}_{j}$ represent the average values of the reair and imaginary parts of $\alpha_{s}\left(x^{*}\right)$ over the path of integration extending from the separation point to the trailing edge, $e^{*}=\varepsilon\left|x_{5}\right|$ is the distance from the separation point to the trailing edge, $n$ is an integer and $A$ and $\psi$ are the amplitude and phase of the quantity

$$
\begin{aligned}
& A e^{-i \psi}=-\frac{5}{32} \frac{1}{\sqrt{2 \pi i}} \frac{c^{\star^{2}}}{e^{* 3 / 2 \hat{f}^{\prime}(0) b_{0}^{1 / 8}}} \\
& x \frac{\left(a_{a}^{+}-a_{s}^{+}\right) \sinh a_{s}^{+} \Delta_{0}\left(\frac{d \alpha_{s}}{d \Delta}\right)_{0}^{1 / 2} K_{+}\left(-a_{s}^{+}\right) f^{\prime}\left(-a_{s}^{+}, \Delta_{0}\right)}{a_{a}^{+}\left(a_{s}^{+}\right) K_{+}^{1 / 2}(0)}
\end{aligned}
$$

where

$b_{0}=\left(1-\ell^{\star}\right) / \varepsilon=0(1)$. Both $A$ and $\varphi$ are $0(1)$.

Note that when matching asymptotic expansions, it is customary to first consider the leading terms. This would lead to Eq. (44). However more accurate results may be obtained if the exponential terms, the power terms and even terms of 0 (1) were consistently included in the expansion. All such terms were included in Eqs. (51) and (52).

\section{Discussion of the Results}

The preceding analysis is a systematic perturbation about the steady state of the equations governing the motion of a fluid around an airfoil with smooth separation at high Reynolds numbers. We have shown that the unsteady equations have eigensolutions for certain discrete values of the Reynoids and Strouhal numbers which can be calculated from Eqs. (51) and (52). We term these discrete values "resonant conditions". Thus at resonant conditions self-sustainable oscillations of the flow take place. These oscillations are self-sustained by a feedback mechanism whereby a Kelvin-Helmholtz instability wave emanating from the separation point on the airfoil surface propagates downstream and is then diffracted as it interacts with the sharp trailing edge of the airfoil causing acoustic radiation (hydrodynamic pressure field) that propagates upstream and regenerates the initial instability wave.

Crighton and Leppington 18 have shown that the interaction between an acoustic source and an unstable shear layer in the presence of a solid surface with a sharp edge produces a spatially growing Kelvin-Helmhoitz instability wave that propagates along the shear layer. The coupling between the acoustic wave and the instability wave takes place at the surface edge where a Kutta condition is applied. This led to the development of analytical models for shear layer feedback cycles by Mohring 19 and Crighton and Innes 20 for geometries with at least two sharp edges. In the present problem we have used Goldstein's coupling coefficient (Eq. (45)) which accounts for viscous effects. This coupling coefficient is small since it is proportional to $\varepsilon_{0}$. This feature makes it possible in the high frequency limit to match the amplitude of the instability wave with that of the scattered hydrodynamic field when the distance $\ell^{\star} / \varepsilon$ between the two ends of the feedback cycle is much larger than the width $\Delta_{0}$ of the separated region at the sharp trailing edge.

The present theory proposes to explain the experiments of Paterson et al. 1 and Arbey and Bataille's $s^{4}$ by a new mechanism based on the generation of spatially growing Kelvin-Helmholtz instability waves emanating from the separation point on the airfoil surface. Our phase condition (Eq. (52)) has essentially the same form as the empirical formula proposed. 4 For comparison of our resonant conditions with data, we note that both experiments were carried out on NACA 0012 airfoils and that the lowest value for $S$ in the data was about 20 . This implies that for these data $\delta>\varepsilon$ and therefore $\Delta_{0}=0(1)$ or larger. This leads to a simplification where many of the terms in Eq. (53) can be replaced by their asymptotic values for large $\Delta$. Thus, we obtain the following approximate expressions for Eqs. (51) and (52), respectively

$$
\frac{1}{\varepsilon_{0}} \sim \frac{5}{32 \sqrt{2 \pi}} \frac{c^{\star^{2}}}{l^{\star^{3 / 2}} b_{0} 1 / 8 f^{\prime}(0) \mid} \frac{e^{\left(-\bar{a}_{i} l^{\star}-\Delta_{0}\right) S}}{S^{2}}
$$




$$
\begin{aligned}
& \bar{a}_{r}\left(e^{\star}+\Delta_{0}^{\star}\right) S--\frac{\pi}{4}+2 \pi n \text { for } \hat{f}^{\prime}(0)>0 \\
& \bar{a}_{r}\left(\iota^{\star}+\Delta_{0}^{\star}\right) S--\frac{\pi}{4}+(2 n-1) \pi \text { for } \hat{f}^{\prime}(0)<0
\end{aligned}
$$

where $\Delta_{0}^{\star}=\varepsilon \Delta_{0}$. Note that $\iota^{\star}, \Delta_{0}^{\star}$ and $c^{*}$ depend only on the airfoil geometry. To the degree of our approximation (Eqs. (44), (49), and (50)) it is permissible however to evaluate these quantities at the separation point.

The assumption of a separated flow is central to our model. The steady flow characteristics around a NACA 0012 airfoil have been extensively studied. The maximum velocity occurs at $0.14 \mathrm{c}$ from the leading edge with a practically flat variation between 0.10 and $0.15 \mathrm{c}$ (Abbott and von Doenhoff $21, p .321)$. Theoretical and experimental investigation of boundary-layer separation on NACA 0012 airfoils were carried out by McCroskey 7 for a Reynolds number range between $5 \times 10^{4}$ and $2 \times 10^{5}$. At zero angle of attack, the location of the theoretical laminar separation point was $0.70 \mathrm{c}$ from the leading edge.

Our present analysis is based on an asymptotic theory for high Reynolds number. To leading order in Re the steady-state solution is the trigle deck Kirchhoff model of Sychev. II Smith 12,22 studied this model and showed that such a steady-state, solution exists for a bluff body. Cheng and Smith 16 extended the solution to a slender profile and studied the effects of scaling the profile thickness $\delta$ with inverse power of the Reynolds number, Their solution was recently complemented by cheng 23 who considered the closure of the wake and showed that the Brown and Stewarton description 24 involving a Goldstein wake 25 can be approached by Sychev's model as the downstream end of the closed wake moved forward to the trailing edge. We therefore use this model as the asymptotic steady-state solution for large Re. We note that Eq. (44) implies that $s \gg \varepsilon_{0} / 2$. The airfoil then appears as a bluff body vis-a-vis the flow and the separation point will be within a distance $0\left(c_{0} / 2\right)$ of the Brillouin point, However, for a practical Reynolds number range, ef/2 is not very small and the accuracy of the results could be enhanced by determining the position of the separation point to $0(\varepsilon \delta / 2)$.

If $s P$ is the pressure, then using the thin airfoil theory approximation one can show that $\mathrm{P}+\mathrm{iH}_{\mathrm{s}}$ is an analytic function of the complex variable $2^{*}=x^{*}+$ iy*. Following an analysis similar to that of Cheng and Smith 16 we can derive the following expressions for the pressure and free streamline shape

$$
\begin{aligned}
& P=-\frac{1}{\pi}\left(x_{0}^{\star}-x^{\star}\right)^{1 / 2} F\left(x_{0}^{\star}\right) \text { as } x^{\star}+x_{0}^{\star} \\
& H_{s}^{\prime}=\frac{1}{\pi}\left(x^{\star}-x_{0}^{\star}\right)^{1 / 2} \int_{0}^{x_{0}^{\star}} \frac{H_{b}^{\prime}(t)}{\sqrt{x_{0}^{\star}-t\left(x^{\star}-t\right)}} d t x^{\star}>x_{0}^{\star}
\end{aligned}
$$

where

$$
F\left(x_{0}^{\star}\right)=\int_{0}^{x_{0}^{\star}} \frac{H_{b}^{\prime}(t)-H_{b}^{\prime}\left(x_{0}^{*}\right)}{\left(x_{0}^{\star}-t\right)^{3 / 2}} d t-\frac{2 H_{b}^{\prime}\left(x_{0}^{\star}\right)}{\sqrt{x_{0}^{*}}}
$$

The condition for a smooth separation leads to

$$
F\left(x *_{B P}\right)=0 \text {. }
$$

Equation (60) gives the location of the Brillouin point.

On the other hand, the triple deck criterion for separation is

$$
\delta P \sim-(0.44) \sigma^{9 / 8} \varepsilon_{0}^{1 / 2}\left(x_{0}^{\star}-x^{\star}\right)^{1 / 2} \text { as } x^{\star} \rightarrow x_{0}^{\star}
$$

Equating Eqs. (57) and (61) and using Blasius expression for the scaled skin friction $\sigma$, gives an equation for $x_{0}^{*}$ in terms of $e^{*}$ and $\delta$. Figure 5 gives the variation of $x_{0}^{\star}$ versus Re for a NACA 0012 profile. The Briliguin point is located at $\times B P=0.11$ c. For $8 \times 10^{4} \leq$ Re $\leq 2 \times 10^{6}$
which covers the range of the availabTe experimental data, we find

$$
0.35 \leq x_{0}^{\star} \leq 0.50 \text {. }
$$

We first note that the location of the separation point depends weakly on the Reynolds number. Classical calculations of laminar separation are independent of the Reynolds number. Second, the discrepancy between Eq. (62) and McCroskey's calculations can be attributed to the following. (a) The calculation of the position of the laminar separation point always involves a certain degree of uncertainty, particularly in cases where the point of separation is located comparatively far behind the point of minimum pressure (as is the case for a NACA 0012 girfo1l) as shown by the experiments of Schubauer $^{26}$ on elliptical cylinders 27 (p. 203). (b) The methods used to predict the location of the separation point often give the location of Goldstein's singularity 28 which is always located downstream of the separation point.

Since we do not know the magnitude and sign of $f^{\prime}(0)$, we give in Tables I and II the values for $\tilde{S}=S / 2 \pi$, the Strouhal number most comnoniy used in experiments, and if $(0) 1$ calculated from Eqs. (54) to (56) versys $n$ for assumed values of $\mathrm{Re}=10^{5}$ and $5 \times 10^{5}$, respectively. We observe that for $f^{\prime}(0)=0(1)$, as suggested by the theory, $\widetilde{s}$ would have a value between 3 and 5 . These are the values of $\mathcal{S}$ corresponding to the lowest observed frequencles in Arbey and Bataflle's data.

As we examine the resonant conditions we note that there is an infinite set of eigenvalues $s(n)$ and $\operatorname{Re}(n)$ corresponding to all values of the integer $n$. However, as Tables $I$ and II show for the present example only $n=2$ to 4 corresponds to a realistic value of the Reynolds number. For smaller $n$, the Reynolds number will be below the cut-off value for which the eddy disappears and no separation occurs on the airfoll surface. On the other hand, for larger $n$, the Reynolds number will 
be orders of magnitude larger than values where the boundary layer can be laminar.

The experimental data, however, show that for the same Reynolds number there are many discrete tones emitted from the airfoil and that the Strouhal numbers corresponding to these tones satisfy a relationship of the same form as Eq. (52). This suggests that the self-sustainable oscillatory flow phenomenon still exists for larger values of $n$. This would imply changing the equality (Eq. (51)) into

$$
\frac{1}{\varepsilon_{0}} \leq A \varepsilon^{15 / 18} \exp \left\{-\bar{\alpha}_{j} \frac{\ell^{\star}}{\varepsilon}\right\} .
$$

That is the magnitude of the coupling coefficient is larger than what is needed to regenerate the instability wave.

In order to understand the implication of Eq. (63) we consider the present problem as an initial value problem (though we realize we are essentially using the steady state solution) with an external forcing pulse concentrated around a frequency w for which we satisfy the phase matching relationship (Eq. (52)). This external forcing will trigger an instability wave at the airfoil surface separation point, which will grow as it propagates downstream. As it impinges on the sharp trailing edge, the instability wave will be diffracted and will cause a pressure wave which will propagate upstream and will be larger than the initial external forcing. As this larger pressure wave acts on the separation point, it will generate still a larger amplitude instability wave which will be further amplified as the feedback mechanism continues. The flow is therefore linearly unstabie. But as the amplitude of the unsteady motion grows, nonlinear effects become significant and eventually modify the basic flow. However the persistence of the discrete tones at higher Strouha? number indicates weakly nonlinear but globaly stable phenomenas in this case as recently suggested by crighton 29 the phase relationship obtained from linear analysis may remain valid. Matching the phase will therefore prevent any cancellation due to phase difference and will result in a "super resonant" condition for the flow. The spectral evolution and the energy exchange between modes cannot, of course, be predicted by linear analysis.

\section{Acknowledgment}

This work was carried out while the author was the Addison P. Rothrock Distinguished Scientist at NASA Lewis Research Center on sabbatical leave from the University of Notre Dame. The author wishes to thank the National Aeronautics and Space Administration and the Air Force Office of Scientific Research for providing financial support. The author is grateful to $D r$. Marvin Goldstein for his insightful coments and keen observations during the course of this work. He would also like to thank James Pennline and James Guptill for carrying out the numerical computations.

\section{References}

1. Paterson, R. W., Vogt, P. G., Fink, M. R., Munch, C. L., "Vortex Noise of Isolated Airfoils," J. Aircraft, Vol. 10, No. 7, pp. 296-302 (1973).
2. Tam, C. K. W., "Discrete Tones of Isolated Airfoils," J. Acoust. Soc. Am., Vol. 55, No. 6, pp. 1173-1177 (1974).

3. Fink, M. R., "Prediction of Airfoil Tone Frequencies," J. Aircraft, Vol. 12, No. 2, pp. $118-120$ (1975).

4. Arbey, H. and Bataille, J., "Noise Generated by Airfoil Profiles Placed in a Uniform Laminar Flow," J. Fluid Mech., Vol. 134, pp. 33-47 (1983).

5. Goldstein, M. E., "Scattering of Acoustic Waves into Tollmien-Schlichting Waves by Smal Streamwise Variations in Surface Geometry," submitted to the Journal of Fluid Mechanics.

6. Nishioka, M. and Morkovin, M. V., "Experiments on a Mechanism of Boundary-Layer Receptivity to Unsteady Pressure Gradients," to be submitted for publication.

7. McCroskey, W. J., "Measurements of Boundary Layer Transition, Separation and Streamline Direction on Rotating Blades," NASA TN D-6321 (1971).

8. Rockwell, D., "Oscillations of Impinging Shear Layers," AIAA Journal, Vol. 21, No. 5, pp. 645-664 (1983).

9. Goldstein, M. E., "Generation of Instability Waves in Flows Separating from Smooth Surface, J. Fluid Mech., Vol. 145, pp. 71-94 (1984).

10. Atassi, H. M., "Feedback and Resonance in Separated Flows over Airfoils," submitted to the Journal of Fluid Mechanics.

11. Sychev, V. V., "Laminar Separation," Izv. Akad. Nauk SSSR, Mekh. Zhidk. Gaza, No. 3, pp. 47-59 (1972).

12. Smith, F. T., "The Laminar Separation of An Incompressible Fluid Streaming past a Smooth Surface," Proc. R. Soc., London A., Vol. 356, pp. 443-463 (1977).

13. Noble, B., "Methods Based on the Wiener-Hopf Technique for the Solution of Partial Differential Equations," Pergamon Press, P. 15 (1958).

14. Goldstein, M. E., "The Generation of Instability Waves in Flows Separating from Smooth Surfaces. Part II - Brillouin Point Separation," to be submitted for publication.

15. Brown, S. N. and Cheng, H. K., "Correlated Unsteady and Steady Laminar Trailing-Edge Flows," J. Fluid Mech., Vol. 108, pp. 171-183 (1981).

16. Cheng, H. K. and Smith, F. T., "The Influence of Airfoil Thickness and Reynolds Number on Separation," ZAMP, Vol. 33, pp. 151-180 (1982).

17. Brodetsky, S., "Discont inuous Fluid Motion Past Circular and Elliptic Cylinders," Proc. Roy. Soc. A, Vol. 102, pp. 542-553 (1923). 
18. Crighton, D. G. and Leppington, F. G. "Radiation Properties of the Semi-Infinite Vortex Sheet: the Initial Value Problem," J. Fluid Mech., Vol. 64, Pt. 2, pp. 393-414 (1974).

19. Mohring, W., "On Flows With Vortex Sheets and Solid Plates," J. Sound and Vibration, Vol. 38 , pp. 403-412 (1975).

20. Crighton, D. G. and Innes, D., "Analytical Model for Shear-Layer Feedback Cycles," AIAA Paper No. 81-0061 (1981).

21. Abbott, I. H. and von Doenhoff, A. E., "Theory of Wing Sections," Dover (1954).

22. Smith, F. T., "Laminar Flow of an Incompressible Fluid Past a Bluff Body: The Separation, Reattachment, Eddy Properties and Drag," J. Fluid Mech., Vol. 29, Part 1, pp. 171-205 (1979).
23. Cheng, H. K., "Laminar Separation from Airfoils Beyond Trailing Edge Stall," AIAA Paper 84-1612 (1984).

24. Brown, S. and Stewarton, K., "Trailing Edge Stall," J. Fluid Mech., Vol. 42, pp. 561-581 (1970).

25. Goldstein, S., "Concerning Same Solutions of the Boundary Layer Equations in Hydrodynamics," Proc. Camb. Phil. Soc., Vol. 26, p. 1 (1930).

26. Schubauer, G. B., "Airflow in a Separating Laminar Boundary Layer," NACA Rep. No. 527 (1935).

27. Schlicting, H., "Boundary Layer Theory," McGraw Hill (1968).

28. Goldstein, S., "On Laminar Boundary Layer Flow near a Point of Separation," Quant. J. Mech. Appl. Math., Vol. 1, p. 43 (1948).

29. Crighton, D. G., "The Jet Edge-Tone Feedback Cycle," Bulletin of the American Physical Society, Vol. 28, No. 9, p. 1381 (1983). 
TABLE I. - $\widetilde{S}$ and $\left|\hat{f}^{\prime}(0)\right|$ versus $\mathrm{n}$ at $\operatorname{Re}=10^{5}$

\begin{tabular}{|l|l|l|r|r|}
\hline \multirow{2}{*}{$n$} & \multicolumn{2}{|c|}{$f^{\prime}(0)>0$} & \multicolumn{2}{c|}{$\hat{f}^{\prime}(0)<0$} \\
\cline { 2 - 5 } & $\widetilde{S}$ & $\mid \hat{f}^{\prime}(0) l$ & $\tilde{S}$ & \multicolumn{1}{c|}{$\mid \hat{f}^{\prime}(0) ।$} \\
\hline 1 & 1.48 & $4.3 \times 10^{-4}$ & 0.64 & $1.4 \times 10^{-4}$ \\
2 & 3.17 & 0.0027 & 2.33 & $3.0 \times 10^{-3}$ \\
3 & 4.87 & 3.34 & 4.03 & 0.29 \\
4 & 6.57 & 542.25 & 5.72 & 41.63 \\
5 & 8.26 & 97958 & 7.42 & 7305 \\
\hline
\end{tabular}

TABLE II. $-\tilde{s}$ and $\mid f^{\prime}(0)$ | versus $\mathrm{n}$ at $\mathrm{Re}=5 \times 10^{5}$

\begin{tabular}{|l|c|r|r|r|}
\hline \multirow{2}{*}{} & \multicolumn{2}{|c|}{$\hat{f}^{\prime}(0)>0$} & \multicolumn{2}{|c|}{$\hat{f}^{\prime}(0)<0$} \\
\cline { 2 - 5 }$n$ & $\tilde{S}$ & $\mid \hat{f}^{\prime}(0) 1$ & \multicolumn{1}{c|}{$\tilde{S}$} & \multicolumn{1}{|c|}{$\hat{f}^{\prime}(0) \mid$} \\
\hline 1 & 1.28 & $4.3 \times 10^{-4}$ & 0.55 & $1.3 \times 10^{-4}$ \\
2 & 2.74 & 0.032 & 2.01 & $3.2 \times 10^{-3}$ \\
3 & 4.20 & 4.68 & 3.47 & 0.37 \\
4 & 5.66 & 880.24 & 4.93 & 62.75 \\
5 & 7.13 & 197343 & 6.40 & 13247 \\
\hline
\end{tabular}




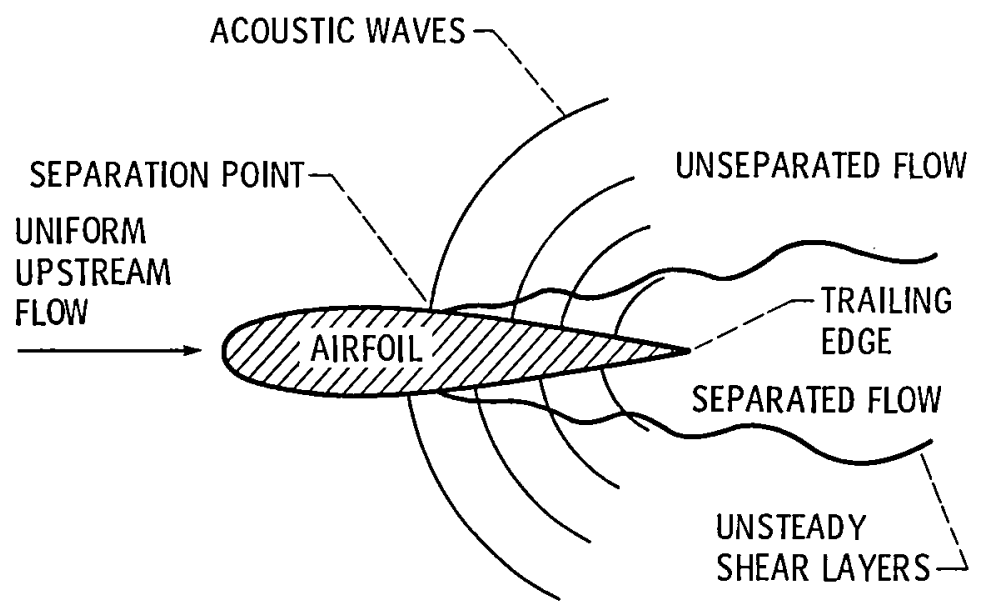

Figure 1. - Unsteady phenomena in separated flow over an airfoil.

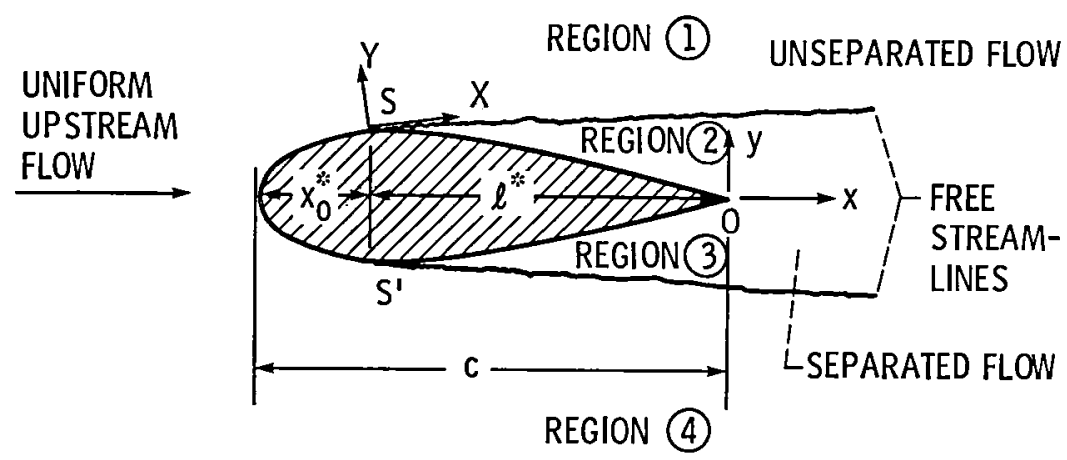

Figure 2. - Configuration of separated flow over an airfoil. 


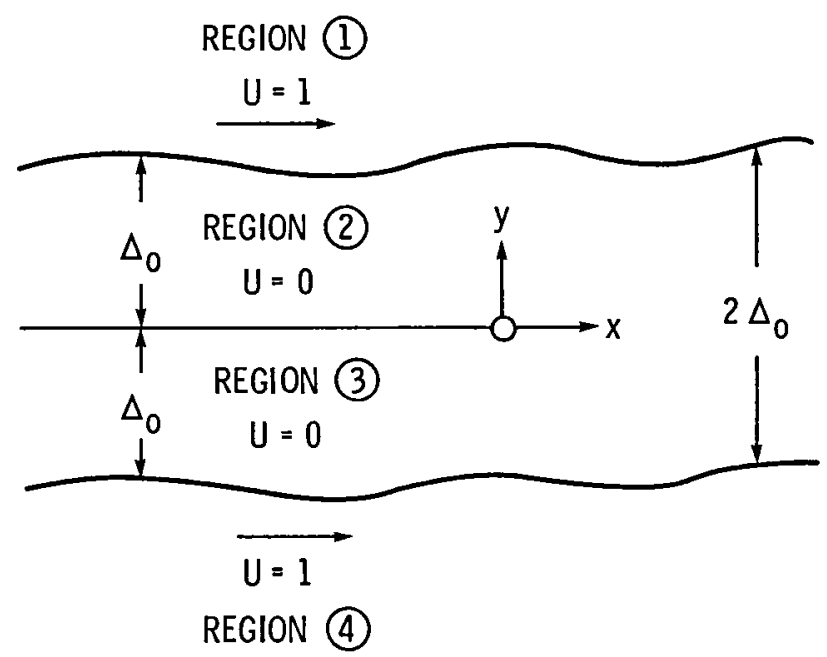

Figure 3. - The scaled trailing edge regions.

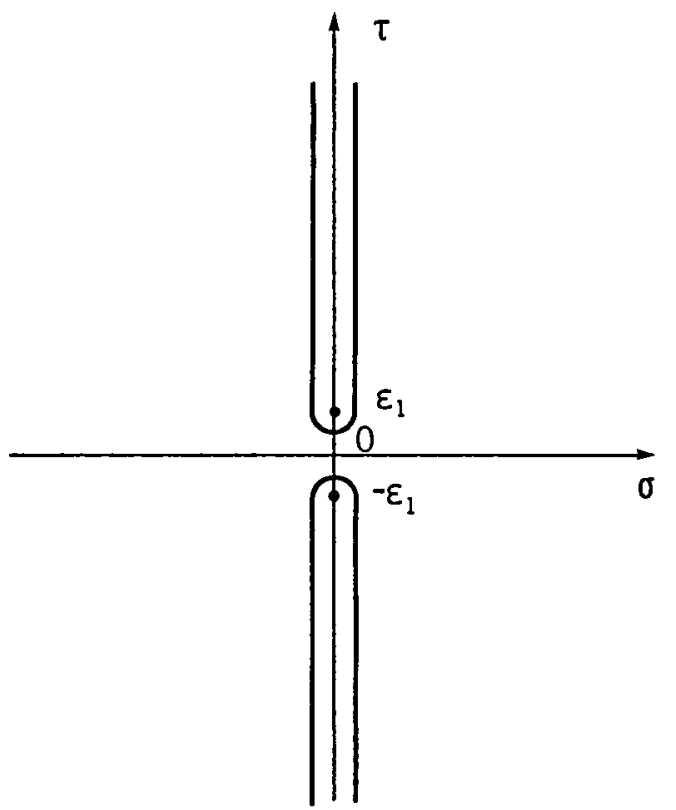

Figure 4. - Branch cut for $\gamma$ in the complex $a$-plane. 


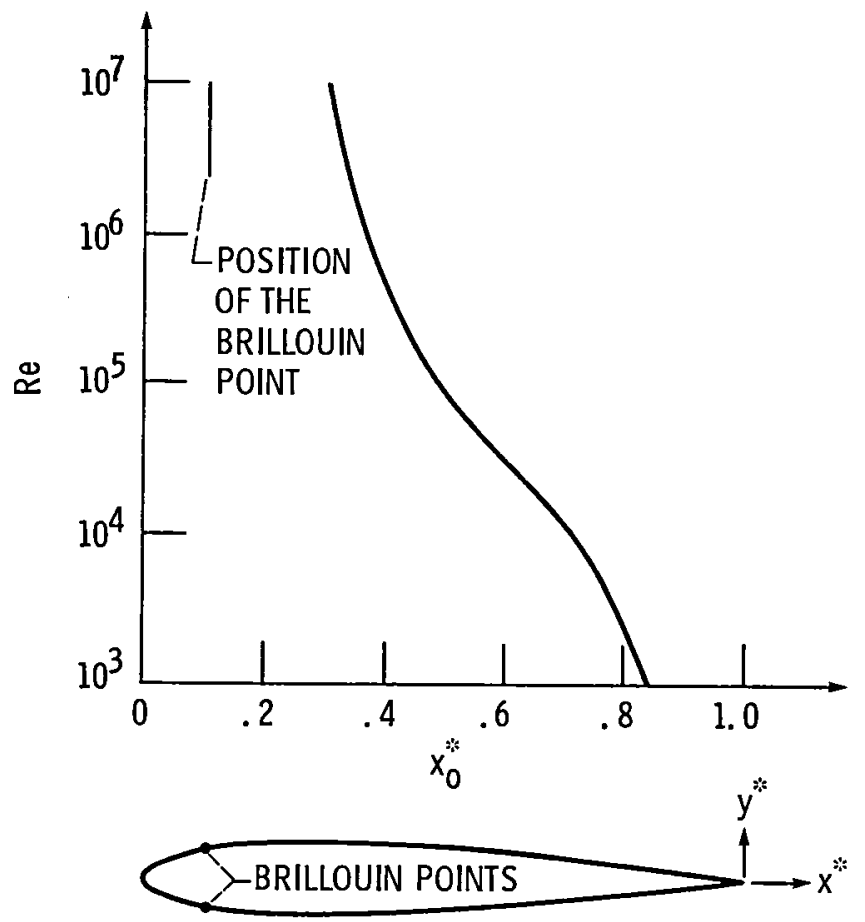

Figure 5. - Position of the separation point versus the Reynolds number for a NACA 0012 airfoil. 


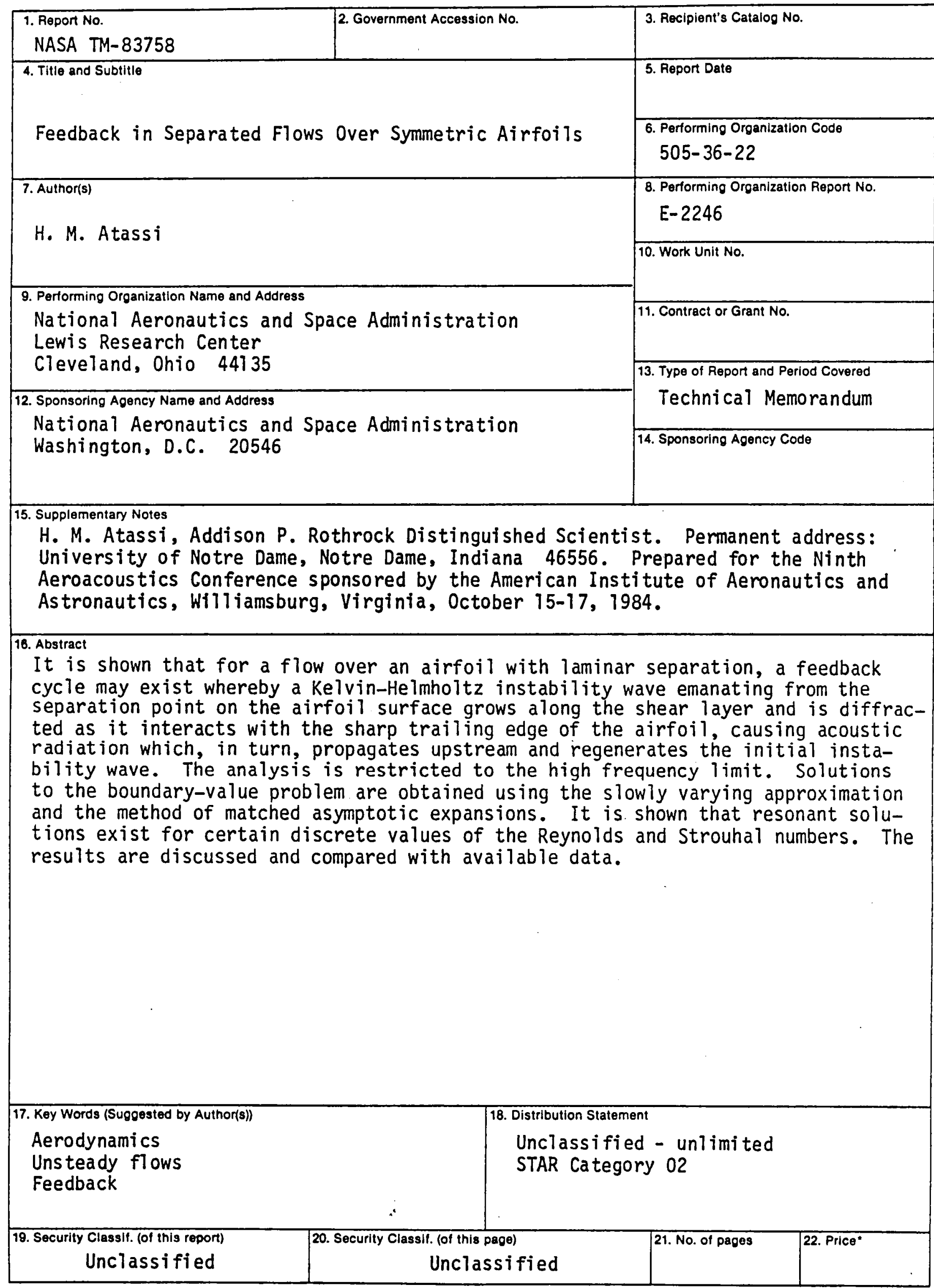

"For sale by the National Technical Information Service, Springfieid, Virginia 22161 
National Aeronautics and Space Admiñistration

Washington, D.C.

20546 .

Official Business

Penalty for Private Use, $\$ 300$
SPECIAL FOURTH CLASS MAIL BOOK

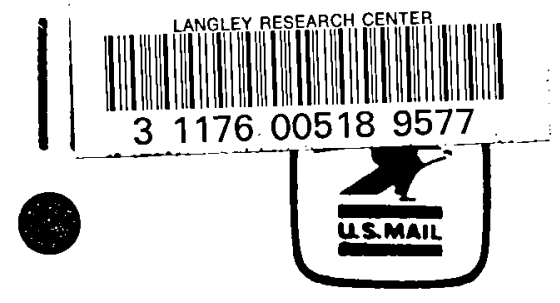

Postage and Fees Paid National Aeronautics and Space Administration NASA-451 